\title{
Simultaneous approximations to $p$-adic numbers and algebraic dependence via multidimensional continued fractions
}

\author{
Nadir Murru ${ }^{1}$ (D) Lea Terracini ${ }^{2}$
}

Received: 9 October 2019 / Accepted: 27 May 2021 / Published online: 17 July 2021

(c) The Author(s) 2021

\begin{abstract}
Unlike the real case, there are not many studies and general techniques for providing simultaneous approximations in the field of $p$-adic numbers $\mathbb{Q}_{p}$. Here, we study the use of multidimensional continued fractions (MCFs) in this context. MCFs were introduced in $\mathbb{R}$ by Jacobi and Perron as a generalization of continued fractions and they have been recently defined also in $\mathbb{Q}_{p}$. We focus on the dimension two and study the quality of the simultaneous approximation to two $p$-adic numbers provided by $p$-adic MCFs, where $p$ is an odd prime. Moreover, given algebraically dependent $p$ adic numbers, we see when infinitely many simultaneous approximations satisfy the same algebraic relation. This also allows to give a condition that ensures the finiteness of the $p$-adic Jacobi-Perron algorithm when it processes some kinds of $\mathbb{Q}$-linearly dependent inputs.
\end{abstract}

Keywords Jacobi-Perron algorithm $\cdot$ Multidimensional continued fractions $\cdot p$-Adic numbers $\cdot$ Simultaneous approximations

Mathematics Subject Classification $11 \mathrm{~J} 61 \cdot 11 \mathrm{~J} 70 \cdot 12 \mathrm{~J} 25$

\section{Introduction}

Continued fractions give a representation for any real number by means of a sequence of integers, providing along the way rational approximations. In particular, they pro-

$\triangle \quad$ Nadir Murru

nadir.murru@unitn.it

Lea Terracini

lea.terracini@unito.it

1 Department of Mathematics, University of Trento, Via Sommarive 14, 38123 Povo, TN, Italy

2 Department of Mathematics G. Peano, University of Torino, Via Carlo Alberto 10, 10123 Torino, Italy 
vide best approximations, i.e., the $n$th convergent of the continued fraction of a real number is closer to it than any other rational number with a smaller or equal denominator. Multidimensional continued fractions (MCFs) are a generalization of classical continued fractions introduced by Jacobi [22] and Perron [34] in an attempt to answer a question posed by Hermite about a possible generalization of the Lagrange theorem for continued fractions to other algebraic irrationalities. A MCF is a representation of a $m$-tuple of real numbers $\left(\alpha_{0}^{(1)}, \ldots, \alpha_{0}^{(m)}\right)$ by means of $m$ sequences of integers $\left(\left(a_{n}^{(1)}\right)_{n \geq 0}, \ldots,\left(a_{n}^{(m)}\right)_{n \geq 0}\right.$ ) (finite or infinite) obtained by the Jacobi-Perron algorithm:

$$
\begin{cases}a_{n}^{(i)}=\left[\alpha_{n}^{(i)}\right], & i=1, \ldots, m, \\ \alpha_{n+1}^{(1)}=\frac{1}{\alpha_{n}^{(m)}-a_{n}^{(m)}}, & n=0,1,2, \ldots, \\ \alpha_{n+1}^{(i)}=\frac{\alpha_{n}^{(i-1)}-a_{n}^{(i-1)}}{\alpha_{n}^{(m)}-a_{n}^{(m)}}, & i=2, \ldots, m,\end{cases}
$$

We shall write

$$
\left(\alpha_{0}^{(1)}, \ldots, \alpha_{0}^{(m)}\right)=\left[\left(a_{0}^{(1)}, a_{1}^{(1)}, \ldots\right), \ldots,\left(a_{0}^{(m)}, a_{1}^{(m)}, \ldots\right)\right]
$$

The Jacobi-Perron algorithm has been widely studied concerning its periodicity and approximation properties. For instance, in $[8,9,26,35]$ the authors provided some classes of algebraic irrationalities whose expansion by the Jacobi-Perron algorithm becomes eventually periodic. In [30], a criterion of periodicity, involving linear recurrence sequences, is given. The periodicity of the Jacobi-Perron algorithm is also related to the study of Pisot numbers [19,20]. Further studies on MCFs can be found in $[2,16,29,41]$.

Continued fractions for $p$-adic numbers were introduced in three different ways [10, $36,37]$ and subsequently studied by several authors like [7,11,13,21]. More recently they have been generalized to higher dimensions. In [31], the authors studied the fundamental properties of MCFs in $\mathbb{Q}_{p}$, focusing on convergence properties and finite expansions, whereas in [32] further properties regarding finiteness and periodicity of the $p$-adic Jacobi-Perron algorithm have been proved.

The study of simultaneous approximations of real numbers is a very important topic in Diophantine approximation; classical and fundamental results can be found in $[3,15,17,18,23]$. Also in the $p$-adic setting simultaneous approximation has been investigated, e.g., in $[1,27,39]$. Some results can also be found regarding simultaneous approximations in $\mathbb{Q}_{p}$, involving a $p$-adic number and its integral powers $[12,28]$. Specific results regarding the case of a $p$-adic number and its square are investigated in $[6,40]$. However, there are no applications of MCFs for providing simultaneous approximations of $p$-adic numbers and for studying the quality of such approximations.

MCFs have been deeply studied in this context for the real case, since they provide simultaneous rational approximations to real numbers. The quality of these simultaneous approximations has been studied in several works, such as [5,14,24,33,38], 
thus, it seems natural to exploit MCFs in $\mathbb{Q}_{p}$ for approaching the problem of constructing simultaneous approximations to $p$-adic numbers. In this paper, we give a first study in this direction and we also investigate the relation between simultaneous approximations and algebraic dependence.

The paper is structured as follows. In Sect. 2, we introduce the notation and we give some basic definitions and properties. Section 3 is devoted to the study of the quality of the simultaneous approximations provided by $p$-adic MCFs. Finally, in Sect. 4, we focus on algebraically dependent pairs of $p$-adic numbers; firstly we find a condition on the quality of approximation under which a sequence of simultaneous rational approximations satisfies the same algebraic relation. Secondly, we apply this result to MCFs and deduce a condition that ensures the finiteness of the $p$-adic Jacobi-Perron algorithm when it processes some kinds of $\mathbb{Q}$-linearly dependent inputs.

\section{Definitions and useful properties}

In the following, we will focus on $p$-adic MCFs of dimension 2, i.e., using the notation of the previous section, we set $m=2$. Most of the results obtained in this paper can be adapted to any dimension $m \geq 2$, but in the general case the notation is very annoying and possibly confusing. Hence, we now recall the $p$-adic Jacobi-Perron algorithm for the case $m=2$; for more details see [31]. From now on, $p$ will be an odd prime number.

Definition 1 The Browkin s-function $s: \mathbb{Q}_{p} \longrightarrow \mathcal{Y}=\mathbb{Z}\left[\frac{1}{p}\right] \cap\left(-\frac{p}{2}, \frac{p}{2}\right)$ is defined by

$$
s(\alpha)=\sum_{j=k}^{0} x_{j} p^{j}
$$

with $\alpha \in \mathbb{Q}_{p}$ written as $\alpha=\sum_{j=k}^{\infty} x_{j} p^{j}, k \in \mathbb{Z}$, and $x_{j} \in \mathbb{Z} \cap\left(-\frac{p}{2}, \frac{p}{2}\right)$.

Given $\alpha, \beta \in \mathbb{Q}_{p}$, we get the corresponding MCF $(\alpha, \beta)=\left[\left(a_{0}, a_{1}, \ldots\right),\left(b_{0}\right.\right.$, $\left.\left.b_{1}, \ldots\right)\right]$ by the following iterative equations:

$$
\left\{\begin{array}{l}
a_{k}=s\left(\alpha_{k}\right), \\
b_{k}=s\left(\beta_{k}\right), \\
\alpha_{k+1}=\frac{1}{\beta_{k}-b_{k}}, \\
\beta_{k+1}=\frac{\alpha_{k}-a_{k}}{\beta_{k}-b_{k}},
\end{array}\right.
$$

for $k=0,1, \ldots$, with $\alpha_{0}=\alpha$ and $\beta_{0}=\beta$. Thus, the complete quotients satisfy the following relations:

$$
\alpha_{k}=a_{k}+\frac{\beta_{k+1}}{\alpha_{k+1}}, \quad \beta_{k}=b_{k}+\frac{1}{\alpha_{k+1}}
$$


and, if the algorithm does not stop, then the initial values are represented by the following MCF:

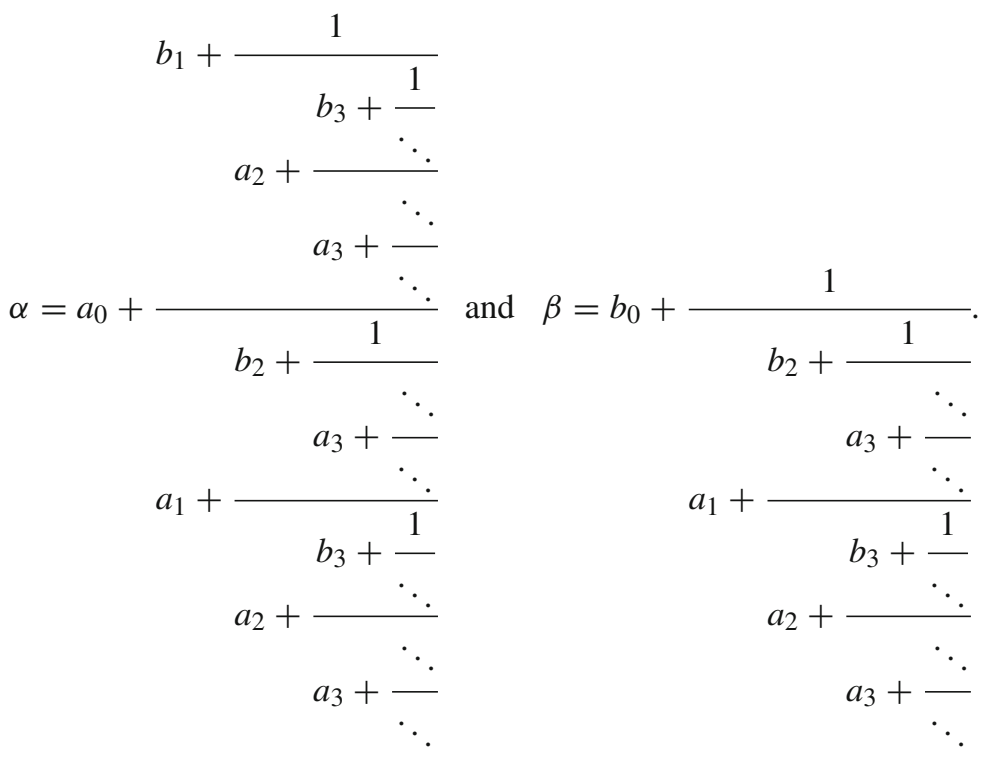

We define the sequences of integers $\left(A_{k}\right)_{k \geq-2},\left(B_{k}\right)_{k \geq-2},\left(C_{k}\right)_{k \geq-2}$ of the numerators and denominators of the convergents, i.e.,

$$
\left[\left(a_{0}, \ldots, a_{n}\right),\left(b_{0}, \ldots, b_{n}\right)\right]=\left(\frac{A_{n}}{C_{n}}, \frac{B_{n}}{C_{n}}\right)=\left(Q_{n}^{\alpha}, Q_{n}^{\beta}\right)
$$

as follows:

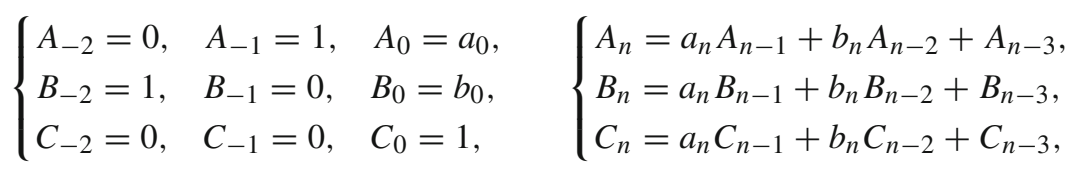

for any $n \geq 1$. Then

$$
\prod_{k=0}^{n}\left(\begin{array}{ccc}
a_{k} & 1 & 0 \\
b_{k} & 0 & 1 \\
1 & 0 & 0
\end{array}\right)=\left(\begin{array}{lll}
A_{n} & A_{n-1} & A_{n-2} \\
B_{n} & B_{n-1} & B_{n-2} \\
C_{n} & C_{n-1} & C_{n-2}
\end{array}\right)
$$

for any $n \geq 0$.

We define the sequences $\left(\tilde{A}_{k}\right)_{k \geq-1}=\left(A_{k} C_{k-1}-A_{k-1} C_{k}\right)$ and $\left(\tilde{B}_{k}\right)_{k \geq-1}=$ $\left(B_{k} C_{k-1}-B_{k-1} C_{k}\right.$ ), arising from the difference between two consecutive conver- 
gents:

$$
Q_{n}^{\alpha}-Q_{n-1}^{\alpha}=\frac{\tilde{A}_{n}}{C_{n} C_{n-1}}, \quad Q_{n}^{\beta}-Q_{n-1}^{\beta}=\frac{\tilde{B}_{n}}{C_{n} C_{n-1}} .
$$

The following relations hold true:

$$
\left\{\begin{array} { l } 
{ \tilde { A } _ { - 1 } = 0 , \quad \tilde { A } _ { 0 } = - 1 , \quad \tilde { A } _ { 1 } = b _ { 1 } , } \\
{ \tilde { B } _ { - 1 } = 0 , \quad \tilde { B } _ { 0 } = 0 , \quad \tilde { B } _ { 1 } = 1 , }
\end{array} \quad \left\{\begin{array}{l}
\tilde{A}_{n}=-b_{n} \tilde{A}_{n-1}-a_{n-1} \tilde{A}_{n-2}+\tilde{A}_{n-3} \\
\tilde{B}_{n}=-b_{n} \tilde{B}_{n-1}-a_{n-1} \tilde{B}_{n-2}+\tilde{B}_{n-3}
\end{array}\right.\right.
$$

for any $n \geq 2$. Indeed,

$$
\begin{aligned}
\tilde{A}_{n}= & A_{n} C_{n-1}-A_{n-1} C_{n} \\
= & \left(a_{n} A_{n-1}+b_{n} A_{n-2}+A_{n-3}\right) C_{n-1}-A_{n-1}\left(a_{n} C_{n-1}+b_{n} C_{n-2}+C_{n-3}\right) \\
= & -b_{n}\left(A_{n-1} C_{n-2}-A_{n-2} C_{n-1}\right)+A_{n-3}\left(a_{n-1} C_{n-2}+b_{n-1} C_{n-3}+C_{n-4}\right) \\
& -C_{n-3}\left(a_{n-1} A_{n-2}+b_{n-1} A_{n-3}+A_{n-4}\right) \\
= & -b_{n} \tilde{A}_{n-1}-a_{n-1} \tilde{A}_{n-2}+\tilde{A}_{n-3}
\end{aligned}
$$

similarly for the $\tilde{B}_{k}$ s. From (4), we have

$$
\begin{aligned}
1= & \operatorname{det}\left(\begin{array}{lll}
A_{n} & A_{n-1} & A_{n-2} \\
B_{n} & B_{n-1} & B_{n-2} \\
C_{n} & C_{n-1} & C_{n-2}
\end{array}\right) \\
= & A_{n} B_{n-1} C_{n-2}-A_{n-1} B_{n} C_{n-2}-A_{n} B_{n-2} C_{n-1}+A_{n-2} B_{n} C_{n-1} \\
& +A_{n-1} B_{n-2} C_{n}-A_{n-2} B_{n-1} C_{n},
\end{aligned}
$$

from which

$$
\frac{1}{C_{n} C_{n-1} C_{n-2}}=\left(Q_{n}^{\alpha}-Q_{n-1}^{\alpha}\right)\left(Q_{n}^{\beta}-Q_{n-2}^{\beta}\right)-\left(Q_{n}^{\beta}-Q_{n-1}^{\beta}\right)\left(Q_{n}^{\alpha}-Q_{n-2}^{\alpha}\right) .
$$

Moreover, since

$$
\alpha=\frac{\alpha_{n} A_{n-1}+\beta_{n} A_{n-2}+A_{n-3}}{\alpha_{n} C_{n-1}+\beta_{n} C_{n-2}+C_{n-3}} \text { and } \beta=\frac{\alpha_{n} B_{n-1}+\beta_{n} B_{n-2}+B_{n-3}}{\alpha_{n} C_{n-1}+\beta_{n} C_{n-2}+C_{n-3}},
$$

we have

$$
\begin{aligned}
& \left(\alpha-Q_{n-1}^{\alpha}\right)\left(\beta-Q_{n-2}^{\beta}\right)-\left(\beta-Q_{n-1}^{\beta}\right)\left(\alpha-Q_{n-2}^{\alpha}\right) \\
& \quad=\frac{1}{C_{n-1} C_{n-2}\left(\alpha_{n} C_{n-1}+\beta_{n} C_{n-2}+C_{n-3}\right)}
\end{aligned}
$$

Let us observe that the previous properties hold for general MCFs, while we now give some specific results regarding only $p$-adic MCFs. In the following we will use 
$v_{p}(\cdot)$ for the $p$-adic valuation, $|\cdot|_{p}$ for the $p$-adic norm and $|\cdot|_{\infty}$ for the Euclidean norm. Moreover, we define

$$
h_{n}=v_{p}\left(\frac{b_{n}}{a_{n}}\right), \quad k_{n}=v_{p}\left(\frac{1}{a_{n}}\right), \quad K_{n}=k_{1}+\cdots+k_{n}
$$

for any $n \geq 1$ and the sequences $\left(V_{k}^{\alpha}\right)_{k \geq-2}=\left(C_{k} \alpha-A_{k}\right)_{k \geq-2},\left(V_{k}^{\beta}\right)_{k \geq-2}=\left(C_{k} \beta-\right.$ $\left.B_{k}\right)_{k \geq-2}$. We recall from [31] the following properties:

- $\left|a_{n}\right|_{p}>1$ and $\left|b_{n}\right|_{p}<\left|a_{n}\right|_{p}$, for any $n \geq 1$;

- $\left|a_{n}\right|_{p}=\left|\alpha_{n}\right|_{p},\left|b_{n}\right|_{p}=\left\{\begin{array}{l}\left|\beta_{n}\right|_{p} \text { if }\left|\beta_{n}\right|_{p} \geq 1 \\ 0 \text { if }\left|\beta_{n}\right|_{p}<1\end{array} \quad\right.$, for any $n \geq 1$;

- $v_{p}\left(C_{n}\right)=-K_{n}$, for any $n \geq 1$;

- $\lim _{n \rightarrow+\infty}\left|V_{n}^{\alpha}\right|_{p}=\lim _{n \rightarrow+\infty}\left|V_{n}^{\beta}\right|_{p}=0$.

\section{The quality of the approximations of $p$-adic MCFs}

In this section, we investigate how well the convergents of a bidimensional continued fraction approach their limit in $\mathbb{Q}_{p}$.

\subsection{The rate of convergence}

In the first instance, we give some results about the rate of convergence of the real sequences $\left|V_{n}^{\alpha}\right|_{p}$ and $\left|V_{n}^{\beta}\right|_{p}$.

Theorem 1 Let $\left[\left(a_{0}, a_{1}, \ldots\right),\left(b_{0}, b_{1}, \ldots\right)\right]$ be the $p$-adic MCF expansion of $(\alpha, \beta) \in$ $\mathbb{Q}_{p}^{2}$, then

$$
v_{p}\left(\alpha-Q_{n}^{\alpha}\right) \geq K_{n}+\left\lfloor\frac{n+2}{2}\right\rfloor, \quad v_{p}\left(\beta-Q_{n}^{\beta}\right) \geq K_{n}+\left\lfloor\frac{n+3}{2}\right\rfloor,
$$

for $n \geq-2$

Proof We will prove by induction that

$$
v_{p}\left(Q_{n+1}^{\alpha}-Q_{n}^{\alpha}\right)=v_{p}\left(\frac{\tilde{A}_{n+1}}{C_{n+1} C_{n}}\right) \geq-v_{p}\left(C_{n}\right)+\left\lfloor\frac{n+2}{2}\right\rfloor,
$$

i.e., we have to prove that

$$
v_{p}\left(\tilde{A}_{n+1}\right) \geq v_{p}\left(C_{n+1}\right)+\left\lfloor\frac{n+2}{2}\right\rfloor
$$


for any $n \geq-2$. We can observe that

$$
v_{p}\left(\tilde{A}_{-1}\right)=v_{p}\left(C_{-1}\right)=\infty,
$$

for $n=-2$, and

$$
v_{p}\left(\tilde{A}_{0}\right)=v_{p}\left(\tilde{C}_{0}\right)=0
$$

for $n=-1$. Moreover,

$$
v_{p}\left(\tilde{A}_{1}\right)=v_{p}\left(b_{1}\right), \quad v_{p}\left(C_{1}\right)=v_{p}\left(a_{1}\right),
$$

for $n=0$, and we know that $v_{p}\left(b_{1}\right)>v_{p}\left(a_{1}\right)$. Now, we proceed by induction, supposing that it is true $v_{p}\left(\tilde{A}_{n}\right) \geq v_{p}\left(C_{n}\right)+\left\lfloor\frac{n+1}{2}\right\rfloor$. Consider

$$
\begin{aligned}
v_{p}\left(\tilde{A}_{n+1}\right) & =v_{p}\left(-b_{n+1} \tilde{A}_{n}-a_{n} \tilde{A}_{n-1}+\tilde{A}_{n-2}\right) \\
& \geq \inf \left\{v_{p}\left(b_{n+1} \tilde{A}_{n}\right), v_{p}\left(a_{n} \tilde{A}_{n-1}\right), v_{p}\left(\tilde{A}_{n-2}\right)\right\},
\end{aligned}
$$

by the inductive hypothesis we have

$$
\begin{aligned}
v_{p}\left(b_{n+1} \tilde{A}_{n}\right) & \geq v_{p}\left(b_{n+1}\right)+v_{p}\left(C_{n}\right)+\left\lfloor\frac{n+1}{2}\right\rfloor \\
& \geq v_{p}\left(a_{n+1}\right)+1+v_{p}\left(C_{n}\right)+\left\lfloor\frac{n+1}{2}\right\rfloor \geq v_{p}\left(C_{n+1}\right)+\left\lfloor\frac{n+2}{2}\right\rfloor .
\end{aligned}
$$

Similarly,

$$
\begin{aligned}
v_{p}\left(a_{n} \tilde{A}_{n-1}\right) & \geq v_{p}\left(a_{n}\right)+v_{p}\left(C_{n-1}\right)+\left\lfloor\frac{n}{2}\right\rfloor \geq v_{p}\left(C_{n+1}\right)+\left\lfloor\frac{n}{2}\right\rfloor+1 \\
& =v_{p}\left(C_{n+1}\right)+\left\lfloor\frac{n+2}{2}\right\rfloor
\end{aligned}
$$

and

$$
\begin{aligned}
v_{p}\left(\tilde{A}_{n-2}\right) & \geq v_{p}\left(C_{n-2}\right)+\left\lfloor\frac{n-1}{2}\right\rfloor \geq v_{p}\left(C_{n+1}\right)+\left\lfloor\frac{n-1}{2}\right\rfloor+3 \\
& \geq v_{p}\left(C_{n+1}\right)+\left\lfloor\frac{n+2}{2}\right\rfloor .
\end{aligned}
$$

Thus, we also have $v_{p}\left(Q_{n+k}^{\alpha}-Q_{n}^{\alpha}\right) \geq-v_{p}\left(C_{n}\right)+\left\lfloor\frac{n+2}{2}\right\rfloor$ and for $k \rightarrow \infty$, we have $v_{p}\left(\alpha-Q_{n}^{\alpha}\right) \geq-v_{p}\left(C_{n}\right)+\left\lfloor\frac{n+2}{2}\right\rfloor$. 
Similar arguments hold for proving $v_{p}\left(Q_{n+1}^{\beta}-Q_{n}^{\beta}\right) \geq-v_{p}\left(C_{n}\right)+\left\lfloor\frac{n+3}{2}\right\rfloor$, i.e., for proving $v_{p}\left(\tilde{B}_{n+1}\right) \geq v_{p}\left(C_{n+1}\right)+\left\lfloor\frac{n+3}{2}\right\rfloor$. We just check the basis of the induction:

$$
v_{p}\left(\tilde{B}_{-1}\right)=\infty, \quad v_{p}\left(\tilde{B}_{0}\right)=\infty, \quad v_{p}\left(\tilde{B}_{1}\right)=0
$$

and

$$
v_{p}\left(C_{-1}\right)=\infty, \quad v_{p}\left(C_{0}\right)=0, \quad v_{p}\left(C_{1}\right)=v_{p}\left(a_{1}\right)<0 .
$$

Corollary 1 Let $\left[\left(a_{0}, a_{1}, \ldots\right),\left(b_{0}, b_{1}, \ldots\right)\right]$ be the $p$-adic MCF expansion of $(\alpha, \beta) \in$ $\mathbb{Q}_{p}^{2}$, then

$$
v_{p}\left(V_{n}^{\alpha}\right) \geq\left\lfloor\frac{n+2}{2}\right\rfloor, \quad v_{p}\left(V_{n}^{\beta}\right) \geq\left\lfloor\frac{n+3}{2}\right\rfloor,
$$

so that

$$
\min \left\{v_{p}\left(V_{n}^{\alpha}\right), v_{p}\left(V_{n}^{\beta}\right)\right\} \geq\left\lfloor\frac{n+2}{2}\right\rfloor=\left\lfloor\frac{n}{2}\right\rfloor+1
$$

Remark 1 In the real case, given $(\alpha, \beta)=\left[\left(a_{0}, a_{1}, \ldots\right),\left(b_{0}, b_{1}, \ldots\right)\right]$ it is well known that

$$
\left|\alpha-\frac{A_{n}}{C_{n}}\right|_{\infty}<\frac{1}{\left|C_{n}\right|_{\infty}}, \quad\left|\beta-\frac{B_{n}}{C_{n}}\right|_{\infty}<\frac{1}{\left|C_{n}\right|_{\infty}}
$$

In the $p$-adic case, a stronger result holds, indeed from the previous theorem we have

$$
\left|\alpha-\frac{A_{n}}{C_{n}}\right|_{p}<\frac{1}{k\left|C_{n}\right|_{p}}, \quad\left|\beta-\frac{B_{n}}{C_{n}}\right|_{p}<\frac{1}{k\left|C_{n}\right|_{p}}
$$

where $k=k(n)$ tends to infinity.

On the other hand formula (6) implies

$$
v_{p}\left(V_{n-1}^{\alpha} V_{n-2}^{\beta}-V_{n-1}^{\beta} V_{n-2}^{\alpha}\right)=K_{n},
$$

which provides an upper bound for the $p$-adic valuation of the $V_{n}$ 's, namely

$$
\min \left\{v_{p}\left(V_{n}^{\alpha}\right), v_{p}\left(V_{n}^{\beta}\right)\right\}+\min \left\{v_{p}\left(V_{n-1}^{\alpha}\right), v_{p}\left(V_{n-1}^{\beta}\right)\right\} \leq K_{n+1} .
$$


This shows that the lower bound for $\min \left\{v_{p}\left(V_{n}^{\alpha}\right), v_{p}\left(V_{n}^{\beta}\right)\right\}$ provided by Corollary 1 is optimal, in the sense that it is reached in some cases:

Example 1 Consider an infinite MCF such that $v_{p}\left(a_{n}\right)=-1$ for every $n \geq 1$. Then $K_{n}=n$ for every $n$, so that by Corollary 1 and formula (7) we get

$$
\min \left\{v_{p}\left(V_{n}^{\alpha}\right), v_{p}\left(V_{n}^{\beta}\right)\right\}+\min \left\{v_{p}\left(V_{n-1}^{\alpha}\right), v_{p}\left(V_{n-1}^{\beta}\right)\right\}=n+1
$$

so that $\min \left\{v_{p}\left(V_{n}^{\alpha}\right), v_{p}\left(V_{n}^{\beta}\right)\right\}=\left\lfloor\frac{n}{2}\right\rfloor+1$ for every $n \geq 1$.

However, in many other cases the bound provided by Corollary 1 can be improved, as stated by the following propositions:

Proposition 1 Let $\left(\ell_{n}\right)_{n \geq 0}$ be a sequence of natural numbers $>0$; put $\ell_{-1}=\ell_{-2}=0$ and define $f(n)=\sum_{j=0}^{n} \ell_{n}$. Let $\left[\left(a_{0}, a_{1}, \ldots\right),\left(b_{0}, b_{1}, \ldots\right)\right]$ be an infinite $p$-adic MCF satisfying $h_{n+1} \geq \ell_{n}, k_{n+1} \geq \ell_{n}+\ell_{n-1}$ for $n \geq 0$. Then for every $n \in \mathbb{N}$

$$
\min \left\{v_{p}\left(V_{n}^{\alpha}\right), v_{p}\left(V_{n}^{\beta}\right)\right\} \geq f(n) .
$$

Proof For $n \geq 1$, either $v_{p}\left(\beta_{n}\right)>0, b_{n}=0, v_{p}\left(\frac{\beta_{n}}{\alpha_{n}}\right)>k_{n}$ or $v_{p}\left(\beta_{n}\right)=v_{p}\left(b_{n}\right) \leq 0$, $v_{p}\left(\frac{\beta_{n}}{\alpha_{n}}\right)=v_{p}\left(\frac{b_{n}}{a_{n}}\right)=h_{n}$. In any case $v_{p}\left(\frac{\beta_{n+1}}{\alpha_{n+1}}\right) \geq \ell_{n}$, for $n \geq 0$. Let $V_{n}$ be either $V_{n}^{\alpha}$ or $V_{n}^{\beta}$. From the formula

$$
V_{n}=-\frac{\beta_{n+1}}{\alpha_{n+1}} V_{n-1}-\frac{1}{\alpha_{n+1}} V_{n-2},
$$

we get for $n \geq 0$

$$
\frac{V_{n}}{p^{f(n)}}=\mu_{n} \frac{V_{n-1}}{p^{f(n-1)}}+v_{n} \frac{V_{n-2}}{p^{f(n-2)}}
$$

where

$$
\mu_{n}=-\frac{\beta_{n+1}}{\alpha_{n+1}} \cdot \frac{1}{p^{\ell_{n}}}, \quad v_{n}=-\frac{1}{\alpha_{n+1}} \cdot \frac{1}{p^{\ell_{n}+\ell_{n-1}}} \in \mathbb{Z}_{p} .
$$

Since $V_{-1}, V_{-2} \in \mathbb{Z}_{p}$ we obtain by induction $\frac{V_{n}}{p^{f(n)}} \in \mathbb{Z}_{p}$.

Corollary 2 Let $f: \mathbb{N} \rightarrow \mathbb{N}$ be any function. There are infinitely many $(\alpha, \beta) \in \mathbb{Q}_{p}^{2}$ satisfying

$$
\min \left\{v_{p}\left(V_{n}^{\alpha}\right), v_{p}\left(V_{n}^{\beta}\right)\right\} \geq f(n)
$$

Proof Of course we can assume $f(n)$ strictly increasing, so that $f(n)=\sum_{j=0}^{n} \ell_{n}$ with $\ell_{n} \in \mathbb{N}, \ell_{n}>0$; the proof follows from Proposition 1 by observing that there are infinitely many $p$-adic MCF satisfying $h_{n+1} \geq \ell_{n}, k_{n+1} \geq \ell_{n}+\ell_{n-1}$ for $n \geq 0$. 
We would like to investigate in which sense and to which extent the approximations given by $p$-adic convergents may be considered "good approximations."Observe that the Browking $s$-function is locally constant, hence so is the function $\mathbb{Q}_{p}^{2} \rightarrow \mathbb{Q}^{2}$ associating to a pair $(\alpha, \beta)$ of its $n$th convergents $\left(Q_{n}^{\alpha}, Q_{n}^{\beta}\right)$ (where this function is defined). Therefore every $(\alpha, \beta) \in \mathbb{Q}_{p}^{2}$ having a MCF of length $\geq n$ has a neighborhood $U$ such that every $\left(\alpha^{\prime}, \beta^{\prime}\right) \in U$ has the same $k$-convergents as $(\alpha, \beta)$ for $k \leq n$. The following proposition will provide an explicit radius for this neighborhood.

Proposition 2 Let $(\alpha, \beta) \in \mathbb{Q}_{p}^{2}$ be such that the associated $\operatorname{MCF}\left[\left(a_{0}, a_{1}, \ldots\right),\left(b_{0}\right.\right.$, $\left.\left.b_{1}, \ldots\right)\right]$ has length $\geq n$. Let $\left(\alpha^{\prime}, \beta^{\prime}\right) \in \mathbb{Q}_{p}^{2}$. If $\max \left\{\left|\alpha-\alpha^{\prime}\right|_{p},\left|\beta-\beta^{\prime}\right|_{p}\right\}<\frac{1}{p^{2 K_{n}}}$, then the MCF $\left[\left(a_{0}^{\prime}, a_{1}^{\prime}, \ldots\right),\left(b_{0}^{\prime}, b_{1}^{\prime}, \ldots\right)\right]$ associated to $\left(\alpha^{\prime}, \beta^{\prime}\right)$ has length $\geq n$ and $a_{i}=a_{i}^{\prime}, b_{i}=b_{i}^{\prime}$, for $i=0, \ldots, n$.

Proof Notice that $\frac{1}{p^{2 K_{n}}}=\frac{1}{\left|C_{n}\right|_{p}^{2}}$. We prove the thesis by induction on $n$. The claim is certainly true for $n=0$, since in general

$$
|x-y|_{p}<1 \Leftrightarrow s(x)=s(y) .
$$

Suppose now $n \geq 1$, and $\max \left\{\left|\alpha-\alpha^{\prime}\right|_{p},\left|\beta-\beta^{\prime}\right|_{p}\right\}<\frac{1}{\left|C_{n+1}\right|_{p}^{2}}$. By the case $n=0$ we have $a_{0}^{\prime}=a_{0}, b_{0}^{\prime}=b_{0}$. Moreover, we observe that our hypothesis implies $\left|\beta-\beta^{\prime}\right|<$ $\frac{1}{\left|a_{1}\right|_{p}}=\left|\beta-b_{0}\right|_{p}$. By the properties of the non-Archimedean norm, we have

$$
\frac{1}{\left|a_{1}^{\prime}\right|_{p}}=\left|\beta^{\prime}-b_{0}\right|_{p}=\max \left\{\left|\beta^{\prime}-\beta\right|_{p},\left|\beta-b_{0}\right|_{p}\right\}=\left|\beta-b_{0}\right|_{p}=\frac{1}{\left|a_{1}\right|_{p}},
$$

so that $\left|a_{1}\right|_{p}=\left|a_{1}^{\prime}\right|_{p}$. We have

$$
\left|\alpha_{1}-\alpha_{1}^{\prime}\right|=\left|\frac{1}{\beta-b_{0}}-\frac{1}{\beta^{\prime}-b_{0}}\right|_{p}=\left|a_{1}\right|_{p}^{2}\left|\beta-\beta^{\prime}\right|_{p}<\prod_{j=2}^{n+1} \frac{1}{\left|a_{j}\right|_{p}^{2}}=\frac{1}{\left|C_{n}^{(1)}\right|_{p}^{2}}
$$

where $C_{n}^{(1)}$ is the $n$th denominator of the convergents of the MCF expansion of $\left(\alpha_{1}, \beta_{1}\right)$. Moreover,

$$
\begin{aligned}
\left|\beta_{1}-\beta_{1}^{\prime}\right|_{p} & =\left|\alpha_{1}\left(\alpha-a_{0}\right)-\alpha_{1}^{\prime}\left(\alpha^{\prime}-a_{0}\right)\right|_{p}=\left|\left(\alpha-a_{0}\right)\left(\alpha_{1}-\alpha_{1}^{\prime}\right)+\alpha_{1}^{\prime}\left(\alpha-\alpha^{\prime}\right)\right|_{p} \\
& \leq \max \left\{\left|\left(\alpha-a_{0}\right)\left(\alpha_{1}-\alpha_{1}^{\prime}\right)\right|,\left|a_{1}\right|_{p}\left|\left(\alpha-\alpha^{\prime}\right)\right|_{p}\right\}<\frac{1}{\left|C_{n}^{(1)}\right|_{p}^{2}} .
\end{aligned}
$$

Thus, by inductive hypothesis we have $a_{i}=a_{i}^{\prime}, b_{i}=b_{i}^{\prime}$ for $i=1, \ldots, n+1$. 
Unfortunately, in general the pair $\left(Q_{n}^{\alpha}, Q_{n}^{\beta}\right)$ does not lie in the $p$-adic ball centered in $(\alpha, \beta)$ and having radius $\frac{1}{p^{2 K_{n}}}$, as Example 1 shows. The next proposition gives a constructive sufficient condition ensuring this property.

Proposition 3 Consider an infinite MCF such that $k_{n+1}>k_{n}+k_{n-1}$ and $h_{n}>k_{n-1}$ for $n \geq 2$. Then for every $n \in \mathbb{N}$, $\max \left\{\left|\alpha-Q_{n}^{\alpha}\right|_{p},\left|\beta-Q_{n}^{\beta}\right|_{p}\right\}<\frac{1}{p^{2 K_{n}}}$.

Proof It is a consequence of Proposition 1 .

\subsection{Diophantine study}

In this section, we want to relate the rate of approximation of the convergents of a $p$-adic MCF to the Euclidean size of its numerators and denominators. First, we give a bound on this size.

Lemma 1 Let $\left(a_{n}\right)_{n \in \mathbb{N}}$ be a sequence of real numbers, such that there exists $m \in \mathbb{N}$, $c_{0}, \ldots, c_{m}$ positive real numbers such that $c_{m}>0$ and

$$
\left|a_{n+m+1}\right|_{\infty}<c_{m}\left|a_{n+m}\right|_{\infty}+c_{m-1}\left|a_{n+m-1}\right|_{\infty}+\cdots+c_{0}\left|a_{n}\right|_{\infty} .
$$

Let $\tilde{x}$ be the (unique, by the Cartesian rule of signs) positive real root of the polynomial

$$
f(X)=X^{m+1}-c_{m} X^{m}-\cdots-c_{1} X-c_{0}
$$

and let $M \geq \max \left\{\left|a_{0}\right|_{\infty}, \frac{\left|a_{1}\right|_{\infty}}{\tilde{x}}, \ldots, \frac{\left|a_{m}\right|_{\infty}}{\tilde{x}^{m}}\right\}$. Then $\left|a_{n}\right|_{\infty} \leq M \tilde{x}^{n}$ for every $n \in \mathbb{N}$.

Proof The proof is straightforward by induction on $n$.

Notice that $f(0)=-c_{0}<0$, so that $\tilde{x}>0$, more precisely

$$
\tilde{x}=c_{m}+\frac{c_{m-1}}{\tilde{x}}+\frac{c_{m-2}}{\tilde{x}^{2}}+\cdots+\frac{c_{0}}{\tilde{x}^{m}},
$$

which implies $c_{m}<\tilde{x}$. Put $C=\sum_{i=0}^{m}\left|c_{i}\right|_{\infty}$, if $C<1$, then $f(1)=1-C>0$, so that $0<\tilde{x}<1$, and we can conclude that $c_{m}<\tilde{x}<1$. In the following, $\tilde{x}_{p}$ will be the real root of the polynomial

$$
X^{3}-\frac{1}{2} X^{2}-\frac{1}{2 p} X-\frac{1}{p^{3}}
$$

so that $\frac{1}{2}<\tilde{x}_{p}<1$ and $\lim _{p \rightarrow \infty} \tilde{x}_{p}=\frac{1}{2}$ in $\mathbb{R}$. By specializing to the case of $p$-adic $M C F$ we can apply the previous Lemma to the sequences $\left(A_{n}\right),\left(B_{n}\right),\left(C_{n}\right)$ as in (3) with $m=2$. Considering that $\left|a_{n}\right|_{\infty},\left|b_{n}\right|_{\infty}<\frac{p}{2}$, for every $n \geq 0$, in this special case the role of the polynomial $f(X)$ of the Lemma is played by

$$
X^{3}-\frac{p}{2} X^{2}-\frac{p}{2} X-1
$$


whose real root is $p \tilde{x}_{p}$. Thus, we obtain the following proposition.

Proposition 4 Given the sequences $\left(A_{n}\right),\left(B_{n}\right),\left(C_{n}\right)$ as in (3), there exists $H>0$ such that

$$
\max \left\{\left|A_{n}\right|_{\infty},\left|B_{n}\right|_{\infty},\left|C_{n}\right|_{\infty}\right\} \leq H\left(p \tilde{x}_{p}\right)^{n}
$$

for every $n \in \mathbb{N}$ and in particular

$$
\max \left\{\left|A_{n}\right|_{\infty},\left|B_{n}\right|_{\infty},\left|C_{n}\right|_{\infty}\right\}=o\left(p^{n}\right)
$$

Proposition 5 Let $\boldsymbol{\alpha}=(\alpha, \beta) \in \mathbb{Q}^{2}$, and write

$$
\alpha=\frac{x_{0}}{z_{0}}, \quad \beta=\frac{y_{0}}{z_{0}}
$$

with $z_{0} \in \mathbb{Z}$ and $x_{0}, y_{0} \in \mathbb{Z}\left[\frac{1}{p}\right]$.

The p-adic Jacobi-Perron algorithm applied to $\alpha$ stops in a number of steps bounded by $-\frac{\log (M)}{\log \left(\tilde{x}_{p}\right)}$ where

$$
\begin{aligned}
M & =\max \left\{\left|z_{0}\right|_{\infty}, \frac{1}{p}\left|y_{0}\right|_{\infty}+\frac{1}{2}\left|z_{0}\right|_{\infty}, \frac{1}{p^{2}}\left|x_{0}\right|_{\infty}+\frac{1}{2 p}\left|y_{0}\right|_{\infty}+\left(\frac{1}{2 p}+\frac{1}{4}\right)\left|z_{0}\right|_{\infty}\right\} \\
& \leq \max \left\{\left|z_{0}\right|_{\infty}, \frac{1}{2}\left(\left|y_{0}\right|_{\infty}+\left|z_{0}\right|_{\infty}\right), \frac{1}{4}\left(\left|x_{0}\right|_{\infty}+\left|y_{0}\right|_{\infty}+\left|z_{0}\right|_{\infty}\right)\right\} \\
& \leq\left|x_{0}\right|_{\infty}+\left|y_{0}\right|_{\infty}+\left|z_{0}\right|_{\infty} \\
& \leq 3 \max \left\{\left|x_{0}\right|_{\infty},\left|y_{0}\right|_{\infty},\left|z_{0}\right|_{\infty}\right\} .
\end{aligned}
$$

Proof The proof is the same as [31, Theorem 5], but we take into account the number of steps. The $p$-adic JP algorithm produces the sequence of complete quotients $\left(\boldsymbol{\alpha}_{\boldsymbol{n}}\right)_{n \geq 0}$, where

$$
\boldsymbol{\alpha}_{\boldsymbol{n}}=\left(\alpha_{n}, \beta_{n}\right) \in \mathbb{Q}^{m}, \quad \alpha_{n}=\frac{x_{n}}{z_{n}}, \quad \beta_{n}=\frac{y_{n}}{z_{n}},
$$

and $x_{n}, y_{n}, z_{n}$ are generated by the following rules:

$$
\left\{\begin{array}{l}
x_{n}=a_{n} z_{n}+y_{n+1}, \\
y_{n}=b_{n} z_{n}+z_{n+1}, \\
z_{n}=x_{n+1}
\end{array}\right.
$$

with $a_{n}, b_{n} \in \mathcal{Y},\left|y_{n+1}\right|_{p},\left|z_{n+1}\right|_{p}<\left|z_{n}\right|_{p}$. Then $\frac{z_{n}}{p^{n}} \in \mathbb{Z}$ and from the formula

$$
z_{n+1}=z_{n-2}-a_{n-1} z_{n-1}-b_{n} z_{n}
$$


we get by Lemma 1

$$
\begin{aligned}
\frac{\left|z_{n+1}\right|_{\infty}}{p^{n+1}} & <\frac{1}{2} \frac{\left|z_{n}\right|_{\infty}}{p^{n}}+\frac{1}{2 p} \frac{\left|z_{n-1}\right|_{\infty}}{p^{n-1}}+\frac{1}{p^{3}} \frac{\left|z_{n-2}\right|_{\infty}}{p^{n-2}} \\
& <M^{\prime} \tilde{x}_{p}^{n}
\end{aligned}
$$

where

$$
M^{\prime}=\max \left\{\frac{\left|z_{2}\right|_{\infty}}{p^{2}}, \frac{\left|z_{1}\right|_{\infty}}{p},\left|z_{0}\right|_{\infty}\right\} .
$$

Then $z_{n+1}=0$ when $\tilde{x}_{p}^{n} \leq \frac{1}{M^{\prime}}$. We have

$$
\begin{aligned}
\frac{\left|z_{1}\right|_{\infty}}{p} & =\frac{1}{p}\left|y_{0}-b_{0} z_{0}\right|_{\infty}<\frac{1}{p}\left|y_{0}\right|_{\infty}+\frac{1}{2}\left|z_{0}\right|_{\infty} \\
\frac{\left|z_{2}\right|_{\infty}}{p^{2}} & =\frac{1}{p^{2}}\left|y_{1}-b_{1} z_{1}\right|_{\infty} \\
& =\frac{1}{p^{2}}\left|\left(x_{0}-a_{0} z_{0}\right)-b_{1}\left(y_{0}-b_{0} z_{0}\right)\right|_{\infty} \\
& <\frac{1}{p^{2}}\left|x_{0}\right|_{\infty}+\frac{1}{2 p}\left|y_{0}\right|_{\infty}+\left(\frac{1}{2 p}+\frac{1}{4}\right)\left|z_{0}\right|_{\infty} .
\end{aligned}
$$

Therefore $M^{\prime}<M$, so that

$$
\begin{aligned}
& z_{n+1}=0 \text { for } \tilde{x}_{p}^{n} \leq \frac{1}{M} \\
& \text { that is for } n \geq-\frac{\log (M)}{\log \left(\tilde{x}_{p}\right)} .
\end{aligned}
$$

Inequalities (12) and (14) are straightforward.

Corollary 3 Let $t, u \in \mathbb{Z}\left[\frac{1}{p}\right]$ and $v \in \mathbb{Z}$ such that the $p$-adic MCF for $\left(\frac{t}{v}, \frac{u}{v}\right)$ has length $\geq n+1$. Then

$$
\max \left\{|t|_{\infty},|u|_{\infty},|v|_{\infty}\right\} \geq \frac{1}{3 \tilde{x}_{p}^{n}}
$$

Proof With the notation of the proof of Proposition 5, we have $z_{n+1} \neq 0$, then the claim follows from by (14) and (15).

Corollary 4 Let $(\alpha, \beta) \in \mathbb{Q}_{p}^{2}$ be a pair having a p-adic MCF expansion of length $\geq n+1$. Then

$$
\max \left\{\left|A_{n}\right|_{\infty},\left|B_{n}\right|_{\infty},\left|C_{n}\right|_{\infty}\right\} \geq \frac{1}{3 p^{K_{n}} \tilde{x}_{p}^{n}}
$$


Proof If we set $t=p^{K_{n}} A_{n}, u=p^{K_{n}} B_{n}, v=p^{K_{n}} C_{n}$, then the hypothesis of Corollary 3 is fulfilled.

The following theorem establishes an explicit lower bound for the Euclidean length of a pair of rational numbers which is a "good approximation"of a $p$-adic pair w.r.t the corresponding $K_{n}$.

Theorem 2 Let $(\alpha, \beta) \in \mathbb{Q}_{p}^{2}$ be a pair having a p-adic MCF expansion of length $\geq n+1$. Let $\left(\frac{t}{v}, \frac{u}{v}\right) \in \mathbb{Q}^{2}$ with $t, u \in \mathbb{Z}\left[\frac{1}{p}\right], v \in \mathbb{Z}$, and assume $\max \left\{\left|\alpha-\frac{t}{v}\right|_{p},\left|\beta-\frac{u}{v}\right|_{p}\right\}<\frac{1}{p^{2 K_{n+1}}}$; then $\max \left\{|t|_{\infty},|u|_{\infty},|v|_{\infty}\right\} \geq \frac{1}{3 \tilde{x}_{p}^{n}}$.

Proof By Proposition 2 the pair $\left(\frac{t}{v}, \frac{u}{v}\right)$ has the same $M C F$ expansion as $(\alpha, \beta)$ up to $n+1$. The claim follows from Corollary 3 .

\section{Results related to algebraic dependence}

\subsection{A p-adic Liouville-type theorem on algebraic dependence}

The quality of rational approximations to real numbers is related to their algebraic dependence. Indeed, if it is possible to find infinitely many good approximations to a $m$-tuple of real numbers, then they are algebraically independent, see, e.g., [4]. Similar results also hold for the $p$-adic numbers [25]. In the following theorem, we prove a new result of this kind and then we apply it to $p$-adic MCFs.

Lemma 2 Let $C$ be a non-zero integer number, then

$$
|C|_{p} \geq \frac{1}{|C|_{\infty}}
$$

Proof The result follows from $p^{v_{p}(C)} \leq|C|_{\infty}$ and $|C|_{p}=\frac{1}{p^{v_{p}(C)}}$.

The following result is a variant of [25, Theorem 3].

Theorem 3 Given $\alpha, \beta \in \mathbb{Q}_{p} \backslash \mathbb{Q}$ such that $F(\alpha, \beta)=0$, for $F(X, Y) \in \mathbb{Z}[X, Y]$ non-zero polynomial with minimal total degree $D$, let $\left(t_{n}\right)_{n \geq 0},\left(u_{n}\right)_{n \geq 0},\left(v_{n}\right)_{n \geq 0}$ be sequences of integers such that $v_{n} \neq 0$ for every $n \in \mathbb{N}$ and

$$
\lim _{n \rightarrow \infty} \frac{u_{n}}{v_{n}}=\alpha, \quad \lim _{n \rightarrow \infty} \frac{t_{n}}{v_{n}}=\beta
$$

in $\mathbb{Q}_{p}$. Consider $M_{n}=\max \left\{\left|t_{n}\right|_{\infty},\left|u_{n}\right|_{\infty},\left|v_{n}\right|_{\infty}\right\}$ and

$$
U_{n}=\max \left\{\left|\alpha-\frac{t_{n}}{v_{n}}\right|_{p},\left|\beta-\frac{u_{n}}{v_{n}}\right|_{p}\right\}
$$


if

$$
\lim _{n \rightarrow \infty} U_{n} \cdot M_{n}^{D}=0
$$

in $\mathbb{R}$, then $F\left(\frac{t_{n}}{v_{n}}, \frac{u_{n}}{v_{n}}\right)=0$ for $n \gg 0$.

Proof We observe that $v_{n}^{D} \cdot F\left(\frac{t_{n}}{v_{n}}, \frac{u_{n}}{v_{n}}\right) \in \mathbb{Z}$ and

$$
\left|v_{n}^{D} \cdot F\left(\frac{t_{n}}{v_{n}}, \frac{u_{n}}{v_{n}}\right)\right|_{\infty} \leq K M_{n}^{D}
$$

where $K$ is the sum of the Euclidean absolute values of the coefficients of $F(X, Y)$. Therefore, if $F\left(\frac{t_{n}}{v_{n}}, \frac{u_{n}}{v_{n}}\right)$ is not zero, we have

$$
\left|F\left(\frac{t_{n}}{v_{n}}, \frac{u_{n}}{v_{n}}\right)\right|_{p} \geq\left|v_{n}^{D} \cdot F\left(\frac{t_{n}}{v_{n}}, \frac{u_{n}}{v_{n}}\right)\right|_{p} \geq \frac{1}{\left|v_{n}^{D} \cdot F\left(\frac{t_{n}}{v_{n}}, \frac{u_{n}}{v_{n}}\right)\right|_{\infty}} \geq \frac{1}{K M_{n}^{D}}
$$

by Lemma 2 and (18). On the other hand, we can write

$$
F(X, Y)=\sum_{i, j} A_{i j}(X-\alpha)^{i}(Y-\beta)^{j}
$$

with $A_{i j} \in \mathbb{Q}_{p}$. We have

$$
A_{10}=\frac{\partial F}{\partial X}(\alpha, \beta), \quad A_{01}=\frac{\partial F}{\partial Y}(\alpha, \beta),
$$

if $A_{10}=0$ and $\frac{\partial F}{\partial X}(X, Y) \neq 0$ then the latter polynomial would give an algebraic dependence relation between $\alpha$ and $\beta$ of total degree $\leq D-1$, therefore $\frac{\partial F}{\partial X}(X, Y)=0$, that is $X$ does not appear in $F(X, Y)$. Analogously $A_{01}=0$ implies that $\frac{\partial F}{\partial Y}(X, Y)=$ 0 , that is $Y$ does not appear in $F(X, Y)$. It follows that if $A_{i j} \neq 0$ for some $i>0$ then $A_{10} \neq 0$; and if $A_{i j} \neq 0$ for some $j>0$ then $A_{01} \neq 0$. Hence, it is easy to see that for every $i, j$ such that $i+j>1$ and $n \gg 0$

$$
\begin{aligned}
& v_{p}\left(A_{i j}\left(\frac{t_{n}}{v_{n}}-\alpha\right)^{i}\left(\frac{u_{n}}{v_{n}}-\beta\right)^{j}\right) \\
& >\min \left\{v_{p}\left(A_{10}\left(\frac{t_{n}}{v_{n}}-\alpha\right)\right), v_{p}\left(A_{01}\left(\frac{u_{n}}{v_{n}}-\beta\right)\right)\right\} .
\end{aligned}
$$


Therefore for $n \gg 0$, we obtain

$$
\begin{aligned}
\left|F\left(\frac{t_{n}}{v_{n}}, \frac{u_{n}}{v_{n}}\right)\right|_{p} & \leq \max _{i j}\left\{\left|A_{i j}\left(\frac{t_{n}}{v_{n}}-\alpha\right)^{i}\left(\frac{u_{n}}{v_{n}}-\beta\right)^{j}\right|\right\} \\
& =\max \left\{\left|A_{01}\left(\frac{t_{n}}{v_{n}}-\alpha\right)\right|_{p},\left|A_{10}\left(\frac{u_{n}}{v_{n}}-\beta\right)\right|_{p}\right\} \leq H \cdot U_{n},
\end{aligned}
$$

for $H=\max \left\{\left|A_{01}\right|_{p},\left|A_{10}\right|_{p}\right\}$. Putting together equations (19) and (20), we get

$$
\operatorname{frac} 1 K M_{n}^{D} \leq\left|F\left(\frac{t_{n}}{v_{n}}, \frac{u_{n}}{v_{n}}\right)\right|_{p} \leq H \cdot U_{n}
$$

for every $n$ such that $F\left(\frac{t_{n}}{v_{n}}, \frac{u_{n}}{v_{n}}\right) \neq 0$. This implies that there exists $C>0$ such that if $F\left(\frac{t_{n}}{v_{n}}, \frac{u_{n}}{v_{n}}\right) \neq 0$ then $U_{n} \cdot M_{n}>C$. Then hypothesis (17) proves the claim.

Remark 2 We shall apply Theorem 3 with

$$
\frac{u_{n}}{v_{n}}=Q_{n}^{\alpha}, \quad \frac{t_{n}}{v_{n}}=Q_{n}^{\beta},
$$

with $u_{n}, t_{n}, v_{n} \in \mathbb{Z}$ coprime. Set $\delta=\max \{0,-v(\alpha),-v(\beta)\}$, then for $n \gg 0$, we have

$$
\left(t_{n}, u_{n}, v_{n}\right)=p^{K_{n}+\delta}\left(A_{n}, B_{n}, C_{n}\right) .
$$

Consequently, if $M_{n}=\max \left\{\left|t_{n}\right|_{\infty},\left|u_{n}\right|_{\infty},\left|v_{n}\right|_{\infty}\right\}$ then by Proposition 4 there exists $H>0$ such that

$$
M_{n} \leq H p^{K_{n}+n} \tilde{x}_{p}^{n}=o\left(p^{n+K_{n}}\right)
$$

\subsection{Some consequences on linear dependence}

We specialize Theorem 3 to the case $D=1$, i.e., when we have linear dependence. In [31], the authors proved that if the $p$-adic Jacobi-Perron algorithm stops in a finite number of steps, then the initial values are $\mathbb{Q}$-linearly dependent. Further results about linear dependence and $p$-adic MCFs can be found in [32], where it is conjectured that if we start the $p$-adic Jacobi-Perron algorithm with a $m$-tuple of $\mathbb{Q}$-linearly dependent numbers, then the algorithm is finite or periodic. Here, exploiting the previous results, we can give a condition that ensures the finiteness of the $p$-adic Jacobi-Perron algorithm when it processes certain $\mathbb{Q}$-linearly dependent inputs.

Theorem 4 Given $\alpha, \beta \in \mathbb{Q}_{p}$, consider

$$
M_{n}=\max \left\{\left|A_{n}\right|_{\infty},\left|B_{n}\right|_{\infty},\left|C_{n}\right|_{\infty}\right\}, \quad U_{n}=\max \left\{\left|\alpha-\frac{A_{n}}{C_{n}}\right|_{p},\left|\beta-\frac{B_{n}}{C_{n}}\right|_{p}\right\}
$$


where $\left(A_{n}\right),\left(B_{n}\right),\left(C_{n}\right)$ are the sequences of numerators and denominators of convergents of the $M C F$ representing $(\alpha, \beta)$. If

$$
\lim _{n \rightarrow \infty} U_{n} \cdot M_{n}=0
$$

then either $\alpha, \beta, 1$ are linearly independent over $\mathbb{Q}$ or the p-adic MCF expansion of $(\alpha, \beta)$ is finite.

Proof Assume that the $p$-adic MCF for $(\alpha, \beta)$ is not finite, then the sequence $\left(Q_{n}^{\alpha}, Q_{n}^{\beta}\right)$ $p$-adically converges to $(\alpha, \beta)$ by [31, Proposition 3] and $(\alpha, \beta) \notin \mathbb{Q}^{2}$, by Proposition 5. Suppose that $A \alpha+B \beta+C=0$ for some $A, B, C \in \mathbb{Q}$ not all zero. We define the sequence $S_{n}=A A_{n-1}+B B_{n-1}+C C_{n-1}$; Theorem 3 implies that $S_{n}=0$ for $n$ sufficiently large. Furthermore, it is straightforward to see that $S_{n}=A V_{n-1}^{\alpha}+B V_{n-1}^{\beta}$ (see also [32]) and by Corollary 1 we should have $V_{n}^{\alpha}=V_{n}^{\beta}=0$, which is a contradiction, as $(\alpha, \beta) \notin \mathbb{Q}^{2}$, since we have supposed the MCF for $(\alpha, \beta)$ is not finite.

Remark 3 Theorem 4 is an improvement of a result implicitly contained in [32, Proposition 10], namely that if $(1, \alpha, \beta)$ are linearly dependent over $\mathbb{Q}$ and there is a constant $K>0$ such that

$$
\max \left\{\left|V_{n}^{\alpha}\right|_{p},\left|V_{n}^{\beta}\right|_{p}\right\} \leq \frac{K}{p^{n}},
$$

then the $p$-adic Jacobi-Perron algorithm stops in finitely many steps when applied to $(\alpha, \beta)$. In fact (22) implies

$$
U_{n} \cdot p^{K_{n}} \leq \frac{K}{p^{n}}
$$

so that, by (21),

$$
U_{n} \cdot M_{n} \leq H \tilde{x}_{p}^{n} U_{n} p^{K_{n}+n} \leq K H \tilde{x}_{p}^{n} \stackrel{\infty}{\rightarrow} 0 \text { for } n \rightarrow \infty
$$

\subsection{A class of fast convergent $p$-adic MCFs}

Finally, we see some conditions on the partial quotients that produce MCFs converging to algebraically independent numbers or having convergents that satisfy an algebraic relation.

Lemma 3 Given a $\operatorname{MCF}\left[\left(a_{0}, a_{1}, \ldots\right),\left(b_{0}, b_{1}, \ldots\right)\right]$ such that

$$
\begin{aligned}
& k_{n+1} \geq(D-1)\left(k_{n}+k_{n-1}\right)+2 D ; \text { and } \\
& h_{n+1} \geq(D-1) k_{n}+D
\end{aligned}
$$

for $n \gg 0$ and $D \geq 1$, then there exists $C \in \mathbb{R}$ such that

$$
\min \left\{v_{p}\left(V_{n}^{\alpha}\right), v_{p}\left(V_{n}^{\beta}\right)\right\} \geq(D-1) K_{n}+D n+C \quad \text { for } n \in \mathbb{N} .
$$


Proof The argument is the same as in the proof of Proposition 1. In fact, if conditions (23) and (24) hold for every $n \geq 0$ then the claim directly follows from Proposition 1 by putting $\ell_{n}=(D-1) k_{n}+\bar{D}$. In any case hypotheses (23) and (24) imply that

$$
v_{p}\left(\frac{\beta_{n+1}}{\alpha_{n+1}}\right) \geq(D-1) k_{n}+D \text { for } n \gg 0 .
$$

Let $V_{n}$ be one of $V_{n}^{\alpha}, V_{n}^{\beta}$. From the formula (8) we get for $n \gg 0$

$$
\begin{aligned}
& \frac{V_{n}}{p^{(D-1) K_{n}+D n}}=\mu_{n} \frac{V_{n-1}}{p^{(D-1) K_{n-1}+D(n-1)}}+v_{n} \frac{V_{n-2}}{p^{(D-2) K_{n-1}+D(n-2)}}, \\
& \mu_{n}=-\frac{\beta_{n+1}}{\alpha_{n+1}} \frac{1}{p^{(D-1) k_{n}+D}}, \quad v_{n}=-\frac{1}{\alpha_{n+1}} \frac{1}{p^{(D-1)\left(k_{n}+k_{n-1}\right)+2 D}} .
\end{aligned}
$$

By (23) and (25) there exists $n_{0}$ such that $\mu_{n}, v_{n} \in \mathbb{Z}_{p}$ for $n>n_{0}$. Then

$$
\left|\frac{V_{n}}{p^{(D-1) K_{n}+D n}}\right|_{p} \leq \max \left\{\left|\frac{V_{i}}{p^{(D-1) K_{i}+D i}}\right|_{p}, i=0, \ldots, n_{0}\right\} \text { for } n>n_{0}
$$

so that the claim follows by setting

$$
C=\min \left\{v_{p}\left(\frac{V_{i}^{\alpha}}{p^{(D-1) K_{i}+D i}}\right), v_{p}\left(\frac{V_{i}^{\beta}}{p^{(D-1) K_{i}+D i}}\right), i=0, \ldots, n_{0}\right\} .
$$

Theorem 5 Assume that $\alpha, \beta$ are algebraically dependent and let $F(X, Y) \in \mathbb{Q}[X, Y]$ be a non-zero polynomial of minimum total degree $D$ such that $F(\alpha, \beta)=0$. If the MCF expansion of $(\alpha, \beta)$ satisfies conditions (23) and (24), then $F\left(Q_{n}^{\alpha}, Q_{n}^{\beta}\right)=0$ for $n \gg 0$.

Proof Let $M_{n}, U_{n}$ be as in Theorem 3. For $n \gg 0$ and a suitable constant $C>0$ we have

$$
\begin{aligned}
U_{n} & =\frac{1}{p^{K_{n}}} \max \left\{\left|V_{n}^{\alpha}\right|_{p},\left|V_{n}^{\beta}\right|_{p}\right\} \\
& \leq \frac{C}{p^{D\left(K_{n}+n\right)}} \text { by Lemma } 3
\end{aligned}
$$

so that $\lim _{n \rightarrow \infty} U_{n} \cdot M_{n}=0$ by (21). Then the claim follows from Theorem 3 .

Theorem 6 Given $(\alpha, \beta)=\left[\left(a_{0}, a_{1}, \ldots\right),\left(b_{0}, b_{1}, \ldots\right)\right]$ such that

$$
\lim _{n \rightarrow \infty} \frac{k_{n}}{k_{n-1}+k_{n-2}}=\infty, \quad \lim _{n \rightarrow \infty} \frac{h_{n}}{k_{n-1}}=\infty
$$


in $\mathbb{R}$, then either $\alpha, \beta$ are algebraically independent or there exists a non-zero polynomial $F(X, Y) \in \mathbb{Q}[X, Y]$ such that $F\left(Q_{n}^{\alpha}, Q_{n}^{\beta}\right)=0$ for $n \gg 0$.

Proof Let $D>0$. For $n \gg_{D} 0$ we have

$$
\begin{aligned}
& k_{n} \geq D\left(k_{n-1}+k_{n-2}\right) \geq(D-1) D\left(k_{n-1}+k_{n-2}\right)+2 D, \text { and } \\
& h_{n} \geq D k_{n-1} \geq(D-1) k_{n-1}+D .
\end{aligned}
$$

Then the claim follows from Theorem 5.

Remark 4 By Faltings theorem, an algebraic curve having infinitely many rational points must have genus 0 or 1 . This is a strong condition on polynomials $F(X, Y) \in$ $\mathbb{Q}[X, Y]$ such that $F\left(Q_{n}^{\alpha}, Q_{n}^{\beta}\right)=0$, for $n \gg 0$.

Funding Open access funding provided by Universitá degli Studi di Trento within the CRUI-CARE Agreement.

Open Access This article is licensed under a Creative Commons Attribution 4.0 International License, which permits use, sharing, adaptation, distribution and reproduction in any medium or format, as long as you give appropriate credit to the original author(s) and the source, provide a link to the Creative Commons licence, and indicate if changes were made. The images or other third party material in this article are included in the article's Creative Commons licence, unless indicated otherwise in a credit line to the material. If material is not included in the article's Creative Commons licence and your intended use is not permitted by statutory regulation or exceeds the permitted use, you will need to obtain permission directly from the copyright holder. To view a copy of this licence, visit http://creativecommons.org/licenses/by/4.0/.

\section{References}

1. Abercrombie, A.G.: The Hausdorff dimension of some exceptional sets of $p$-adic integer matrices. J. Number Theory 53, 311-341 (1995)

2. Adam, B., Rhin, G.: Periodic Jacobi-Perron expansions associated with a unit. J. de Théorie des Nombres de Bordeaux 23, 527-539 (2011)

3. Adams, W.W.: Simultaneous Diophantine approximations and cubic irrationals. Pac. J. Math. 30, 1-14 (1969)

4. Adams, W.W.: The algebraic independence of certain Liouville continued fractions. Proc. Am. Math. Soc. 95, 512-516 (1985)

5. Baldwin, P.: A convergence exponent for multidimensional continued fraction algorithm. J. Stat. Phys. 66, 1507-1526 (1992)

6. Bugeaud, Y.: On simultaneous uniform approximation to a $p$-adic number and its square. Proc. Am. Math. Soc. 138, 3821-3826 (2010)

7. Belhadef, R., Esbelin, H.A., Zerzaihi, T.: Transcendence of Thue-Morse p-adic continued fractions. Medit. J. Math. 13, 1429-1434 (2016)

8. Bernstein, L.: New infinite classes of periodic Jacobi-Perron algorithms. Pac. J. Math. 16, 439-469 (1965)

9. Bernstein, L.: The Jacobi-Perron Algorithm-Its Theory and Application. Lecture Notes in Mathematics, vol. 207. Springer, Berlin (1971)

10. Browkin, J.: Continued fractions in local fields I. Demonstratio Math. 11, 67-82 (1978)

11. Browkin, J.: Continued fractions in local fields II. Math. Comput. 70, 1281-1292 (2000)

12. Budarina, N., Bugeaud, Y., Dickinson, D., O’Donnell, H.: On simultaneous rational approximation to a $p$-adic number and its integral powers. Proc. Edinb. Math. Soc. 54, 599-612 (2011)

13. Capuano, L., Veneziano, F., Zannier, U.: An effective criterion for periodicity of 1-adic continued fractions. Math. Comput. 88, 1851-1882 (2019) 
14. Chevallier, N.: Best simultaneous approximations and multidimensional continued fraction expansion. Mosc. J. Comb. Number Theory 3, 3-56 (2013)

15. Cusick, T.W.: Formulas for some Diophantine approximation constants II. Acta Arithmetica 26, 117128 (1974)

16. Dasaratha, K., Flapan, L., Garrity, T., Lee, C., Mihaila, C., Neumann-Chun, N., Peluse, S., Stoffregen, M.: Cubic irrationals and periodicity via a family of multi-dimensional continued fraction algorithms. Monatsh. Math. 174(4), 549-566 (2013)

17. Davenport, H.: Simultaneous Diophantine approximation. Proc. Lond. Math. Soc. S3-2, 406-416 (1952)

18. Davenport, H., Mahler, K.: Simultaneous Diophantine approximation. Duke Math. J. 13, 105-111 (1946)

19. Dubois, E., Farhane, A., Paysant-Le Roux, R.: Algorithme de Jacobi Perron: nombres de Pisot, interrumptions et independance lineaire. Ann. Sci. Math. Que. 28, 89-92 (2004)

20. Dubois, E., Farhane, A., Paysant-Le Roux, R.: The Jacobi-Perron algorithm and Pisot numbers. Acta Arithmetica 111, 269-275 (2004)

21. Hirsh, J., Washington, L.C.: p-adic continued fractions. Ramanujan J. 25, 389-403 (2011)

22. Jacobi, C.G.J.: Ges werke VI, pp. 385-426. Academy Press, Berlin (1891)

23. Lagarias, J.C.: Best simultaneous Diophantine approximations. I. Growth rates of best approximations denominators. Trans. Am. Math. Soc. 272, 545-554 (1982)

24. Lagarias, J.C., Hill, M.: The quality of the Diophantine approximations found by the Jacobi-Perron algorithm and related algortihms. Mh. Math. 115, 299-328 (1993)

25. Laohakosol, V., Ubolsri, P.: p-Adic continued fractions of Liouville type. Proc. Am. Math. Soc. 101, 403-410 (1987)

26. Levesque, C., Rhin, G.: Two families of periodic Jacobi Algorithms with period lengths going to infinity. J. Number Theory 37, 173-180 (1991)

27. Lutz, E.: Sur les approximations diophantiennes linéaires $P$-adiques, Actualités Sci. Ind., no. 1224, Hermann \& Cie, Paris (1955)

28. Mahler, K.: Uber eine Klasseneinteilung der P-adischen Zahlen. Mathematica (Zutphen) 3B, 177-185 (1935)

29. Murru, N.: On the periodic writing of cubic irrationals and a generalization of Rédei functions. Int. J. Number Theory 11, 779-799 (2015)

30. Murru, N.: Linear recurrence sequences and periodicity of multidimensional continued fractions. Ramanujan J. 44, 115-124 (2017)

31. Murru, N., Terracini, L.: On p-adic multidimensional continued fractions. Math. Comput. 88, 29132934 (2019)

32. Murru, N., Terracini, L.: On the finiteness and periodicity of the p-adic Jacobi-Perron algorithm. arXiv: 1901.04922

33. Paley, R.E.A.C., Ursell, H.D.: Continued fractions in several dimensions. Proc. Camb. Philos. Soc. 26, 127-144 (1930)

34. Perron, O.: Grundlagen fur eine theorie des Jacobischen kettenbruch algorithmus. Math. Ann. 64, 1-76 (1907)

35. Raju, N.S.: Periodic Jacobi-Perron algorithms and fundamental units. Pac. J. Math. 64, 241-251 (1976)

36. Ruban, A.: Certain metric properties of the p-adic numbers. Sibirsk Math. Z. 11, 222-227 (1970)

37. Schneider, T.: Uber $p$-adische Kettenbruche. Symposia Mathematica 4, 181-189 (1968/69)

38. Schweiger, F.: Some remarks on Diophantine approximation by the Jacobi-Perron algorithm. Acta Arithmetica 133, 209-219 (2008)

39. Sprindžuk, V. G.: Mahler's Problem in Metric Number Theory. Translated from the Russian by B. Volkmann. Translations of Mathematical Monographs, vol. 25, American Mathematical Society, Providence, RI (1969)

40. Teulié, O.: Approximation d'un nombre p-adique par des nombres algébriques. Acta Arithmetica 102, 137-155 (2002)

41. Zhuralev, V.G.: Periodic karyon expansions of cubic irrationals in continued fractions. Proc. Steklov Inst. Math. 296, 36-60 (2017)

Publisher's Note Springer Nature remains neutral with regard to jurisdictional claims in published maps and institutional affiliations. 